\title{
Combined use of ursodeoxycholic acid and bosentan prevents liver toxicity caused by endothelin receptor antagonist bosentan monotherapy: two case reports
}

\author{
Tomoki Ito*, Yoshio Ozaki, Yonsu Son, Tohru Nishizawa, Hideki Amuro, Akihiro Tanaka, Takeshi Tamaki \\ and Shosaku Nomura
}

\begin{abstract}
Introduction: Pulmonary arterial hypertension is a fatal disease characterized by progressive remodeling of the pulmonary arteries and an increase in pulmonary vascular resistance. Up to 50\% of patients with systemic sclerosis have pulmonary arterial hypertension, which significantly affects the prognosis. The endothelin receptor antagonist bosentan is used for the treatment of pulmonary arterial hypertension and shows a great beneficial effect. However, the most frequent side effect of bosentan is liver toxicity, which often requires dose reduction and discontinuation.

Case presentation: We report two cases (a 64-year-old Japanese woman and a 69-year old Japanese woman) of systemic sclerosis, both with severe Raynaud's phenomenon and pulmonary arterial hypertension. Both patients had initially received bosentan monotherapy, which caused liver toxicity as indicated by increased levels of alanine aminotransferase, alkaline phosphatase, and gamma-glutamyltransferase. After dose reduction or discontinuation of bosentan, these liver function abnormalities were normalized and the patients subsequently received retreatment with a combination of bosentan and ursodeoxycholic acid. The results of liver function tests did not show any abnormalities after this combination therapy.

Conclusions: These reports suggest the usefulness of ursodeoxycholic acid for preventing liver toxicity caused by bosentan. Thus, the addition of ursodeoxycholic acid to the treatment protocol is expected to be useful when liver toxicity emerges as a side effect of bosentan.
\end{abstract}

Keywords: Bosentan, Liver toxicity, Pulmonary arterial hypertension, Ursodeoxycholic acid

\section{Introduction}

Pulmonary arterial hypertension is a debilitating disease characterized by progressive remodeling of the pulmonary arteries with proliferation of fibrous tissue in the vessel walls, leading to death caused by right ventricular failure. Approximately 15 to $50 \%$ of patients with systemic sclerosis have pulmonary arterial hypertension, which significantly affects the prognosis $[1,2]$. There is increasing evidence that endothelin-1 plays a pathogenic role in pulmonary arterial hypertension [3]. Several randomized clinical trials have demonstrated the great efficacy

\footnotetext{
* Correspondence: itot@hirakata.kmu.ac.jp

First Department of Internal Medicine, Kansai Medical University, 2-5-1 Shin-machi, Hirakata City, Osaka 573-1010, Japan
}

of endothelin receptor antagonists in pulmonary arterial hypertension; thus bosentan, as the first endothelin receptor antagonist to be marketed, was approved for the treatment of pulmonary arterial hypertension in the USA and Canada in 2001 by the Food and Drug Administration and in Japan in 2005. Bosentan has been reported to be effective for pulmonary arterial hypertension, establishing its position as a novel treatment $[4,5]$, and has recently been approved for the prevention of digital ulcers in systemic sclerosis in Europe.

The most frequent severe side effect of bosentan is known to be liver toxicity [4-8]. The package insert of bosentan indicates that the incidence of liver toxicity is $14.3 \%$ in Japanese clinical studies and 11\% in US clinical 
studies [9]. The incidence reported from post-marketing surveillance in European clinical studies shows annual increased transaminase levels of $10.1 \%$ in 4623 cases, with interruption of bosentan administration being required in $3.2 \%$ of cases [7]. In addition, a Phase III placebo-controlled study for 16 weeks showed $9 \%$ liver toxicity, which is dose-dependent [5]. The therapeutic options for pulmonary arterial hypertension would be expanded if we could find a means to limit liver toxicity.

In the presence of liver toxicity, a dose reduction or discontinuation of bosentan needs to be considered. Although ursodeoxycholic acid can be efficacious against liver injury in clinical practice, there are no published reports of its usefulness in combating bosentan-induced liver toxicity. The incidence of liver toxicity appears to be decreased when ursodeoxycholic acid is simultaneously started with bosentan, but definite evidence of this efficacy has not previously been established.
Two of our patients showed liver toxicity after initial bosentan monotherapy but not with the subsequent addition of ursodeoxycholic acid to their treatment protocol. This is the first report that suggests the usefulness of ursodeoxycholic acid combination therapy to prevent liver toxicity caused by bosentan.

\section{Case presentations}

\section{Case 1}

A 64-year old Japanese woman who presented with edema around her fingers and forearm followed by rapid development of skin sclerosis was diagnosed with systemic sclerosis. She was anti-topoisomerase I (Scl-70) antibody positive and had $35 \mathrm{mmHg}$ of estimated right ventricular pressure, as shown by echocardiography in 2006. She was prescribed prednisolone at $7.5 \mathrm{mg} /$ day. She was negative for both hepatitis B surface antigen (HBs Ag) and hepatitis C virus antibody (HCV Ab). In 2007, dyspnea at exertion and

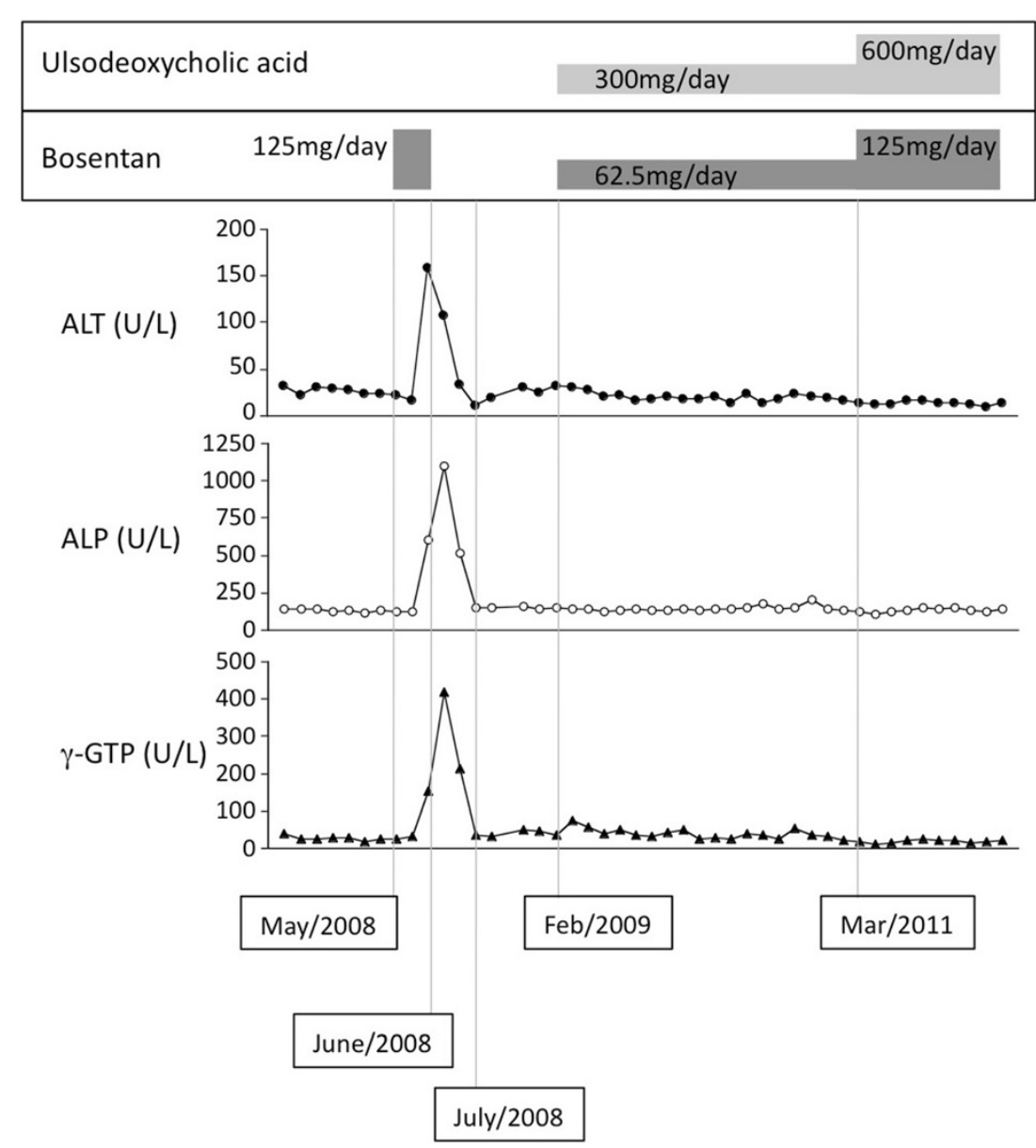

Figure 1 Clinical course of Case 1. Bosentan monotherapy was started in May 2008 at 125mg/day, and after a month, liver function abnormalities appeared. Abnormal results of liver function tests were normalized within a month after the discontinuation of bosentan. In February 2009, combination therapy of bosentan $(62.5 \mathrm{mg} /$ day) and ursodeoxycholic acid $(300 \mathrm{mg} /$ day) was started. There were no subsequent abnormal results of liver function tests, and the dose of both ursodeoxycholic acid and bosentan were increased to 600mg/day and $125 \mathrm{mg} /$ day from March 2011, respectively. No abnormal results of liver function tests were observed. Abbreviations: ALP, alkaline phosphatase; ALT, alanine aminotransferase; GGT, gamma-glutamyltransferase. 
severe Raynaud's phenomenon were confirmed. The findings of a chest computed tomography scan showed bilateral pulmonary fibrotic lesion and an elevated KL-6 level of $4360 \mathrm{U} / \mathrm{mL}$; she was diagnosed with pulmonary fibrosis. In addition, echocardiography results showed an elevation of her estimated right ventricular pressure to $50 \mathrm{mmHg}$; she was therefore clinically diagnosed with pulmonary arterial hypertension (World Health Organization, WHO, functional class III) associated with connective tissue diseasederived pulmonary fibrosis.

After bosentan treatment was initiated in May 2008 at $125 \mathrm{mg}$ /day, dyspnea at exertion improved and her 6 -minute walking distance was increased by $40 \mathrm{~m}$ from $332 \mathrm{~m}$; however, the results of blood tests indicated liver function abnormalities: increased levels of alanine aminotransferase (ALT; 4.5 times the normal upper limit), alkaline phosphatase (ALP; 3.2 times the normal upper limit), and gamma-glutamyltransferase (GGT; 6.4 times the normal upper limit; Figure 1). No other additional drugs were administered during the 2 months before and after the bosentan administration. An ultrasound examination of her liver revealed no morphologic abnormality. The abnormal levels revealed in the liver function tests were normalized soon after the discontinuation of bosentan; however, dyspnea at exertion gradually deteriorated, and therefore bosentan $62.5 \mathrm{mg} /$ day and ursodeoxycholic acid 300mg/day were started simultaneously from February 2009. The results of her liver function tests did not show any abnormalities even after 2 years of the combination therapy, and the dose of both ursodeoxycholic acid and bosentan were increased to $600 \mathrm{mg} /$ day and $125 \mathrm{mg}$ /day, respectively from March 2011. No abnormal levels were found in the results of her liver function tests during 31 months of follow-up after the dose elevation of bosentan. Bosentan improved her estimated right ventricular pressure to $35 \mathrm{mmHg}$, subjective symptoms from WHO functional class III to II, and Raynaud's phenomenon after bosentan retreatment, and she is currently receiving bosentan as an out-patient.

\section{Case 2}

A 69-year-old Japanese woman who presented severe Raynaud's phenomenon, hardening of the skin on her fingers and joint pain was diagnosed with systemic sclerosis. She was anti-centromere antibody and anti-mitochondria antibody positive. Echocardiography results showed $30 \mathrm{mmHg}$ of estimated right ventricular pressure in 2008. Betamethasone $0.25 \mathrm{mg} /$ day was introduced to control her joint pain. Raynaud's phenomenon and dyspnea at exertion started to deteriorate from April 2011. Imaging findings did not show any abnormality in her lung field but echocardiography indicated an elevation of her estimated right ventricular pressure to $45 \mathrm{mmHg}$, and we thus clinically diagnosed pulmonary arterial hypertension associated with connective tissue disease (WHO functional class III).

Bosentan 125mg/day was started from July 2011, and this resulted in the improvement of breathlessness at exertion; however, after 3 weeks the results of her liver function tests revealed abnormal levels: elevation of ALT (1.9 times the normal upper limit), ALP (2.5 times the normal upper limit), and GGT (3.9 times the normal upper limit; Figure 2). No additional drugs were administered during the 2 months before the bosentan administration, and an ultrasound examination of her liver showed no morphologic abnormality at this time point. She was negative for both HBs Ag and HCV Ab. Bosentan was reduced to $62.5 \mathrm{mg} /$ day and ursodeoxycholic acid was introduced simultaneously at $300 \mathrm{mg} /$ day. Her liver

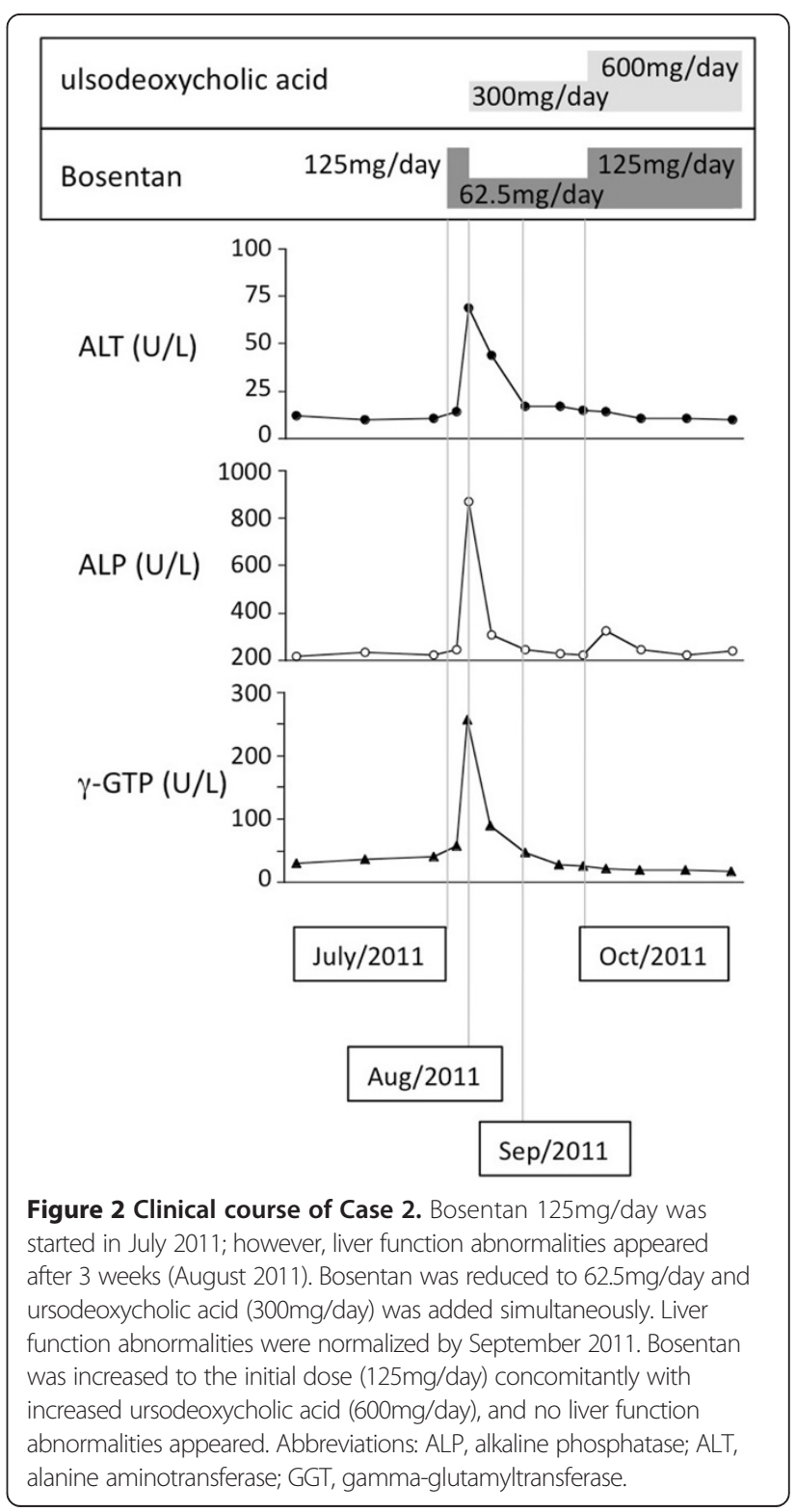


function abnormalities normalized soon after the initiation of the combination therapy. Ursodeoxycholic acid was increased to $600 \mathrm{mg} /$ day and bosentan was increased to the initial dose of $125 \mathrm{mg} /$ day from October 2011. No abnormalities in her liver function were seen during 24 months of follow-up. Bosentan improved her estimated right ventricular pressure to $30 \mathrm{mmHg}$ and her subjective symptoms from WHO functional class III to II after the introduction of bosentan.

\section{Discussion}

The results of the two patients' liver function tests after initial bosentan monotherapy were abnormal, but not when ursodeoxycholic acid was combined as an adjunctive therapy with bosentan. This finding indicates that ursodeoxycholic acid is effective at preventing liver toxicity caused by bosentan, at least in the patients we treated.

Bosentan is mainly metabolized by cytochrome $\mathrm{P} 450$ enzymes CYP3A4 and CYP2C9 of the liver [10] but its pharmacokinetic variability is attributed to liver uptake transporters as well as metabolic enzymes, and the cause of liver injury is suggested to be an inhibition of the bile salt export pump [11]. Since ursodeoxycholic acid has been reported to induce an increased expression of CYP3A [12], the useful effect of ursodeoxycholic acid might be attributable to progression of the CYP3Amediated metabolism of bosentan.

Even though a gradual increase in the dosage of bosentan is recommended, liver toxicity often occurs after its introduction or an increase in the dosage. If severe transaminitis (an over 8-fold increase in ALT/aspartate aminotransferase, AST, of the normal upper limit) occurs, bosentan treatment should be stopped immediately. However, if there is only mild transaminitis (less than an 8-fold increase in ALT/ AST of the normal upper limit), bosentan can be reintroduced after dose reduction or interruption. Abnormal results of liver function tests are, in most cases, normalized after discontinuing bosentan, but we are often reluctant to restart bosentan. Our report suggests that it is, however, worth trying the reintroduction along with ursodeoxycholic acid, at least on the transient mild transaminitis. Pulmonary arterial hypertension still remains an incurable disease with a severe prognosis, and it is therefore important to start treatment with specific and effective drugs. Because bosentan is useful for the treatment of pulmonary arterial hypertension, it is desirable to avoid a dose reduction or discontinuation due to liver intolerance. Although there are alternative drugs (such as another endothelin receptor antagonist, ambrisentan, and phosphodiesterase type 5 inhibitors, sildenafil and tadalafil) with low liver toxicity approved for pulmonary arterial hypertension [13], it remains preferable to have more options available to us. Ursodeoxycholic acid is commonly used because of its safety and relatively low incidence of side effects, and its concomitant administration might help control the side effects of bosentan.

\section{Conclusions}

In summary, the present report suggests that ursodeoxycholic acid has the potential to repress liver toxicity caused by bosentan. Thus, the combined use of oral ursodeoxycholic acid with bosentan is expected to be useful when liver toxicity emerges as a side effect of bosentan monotherapy.

\section{Consent}

Written informed consent was obtained from the patients for publication of this case report and any accompanying images. Copies of the written consents are available for review by the Editor-in-Chief of this journal.

\section{Abbreviations}

ALP: Alkaline phosphatase; ALT: Alanine aminotransferase; AST: Aspartate aminotransferase; GGT: Gamma-glutamyltransferase; HBs Ag: Hepatitis B surface antigen; HCV Ab: Hepatitis C virus antibody; WHO: World Health Organization.

\section{Competing interests}

The authors declare that they have no competing interests.

\section{Authors' contributions}

TI supervised and wrote the manuscript. YO, YS, TN, HA, AT, TT, and SN participated in the patients' therapy and helped to draft the manuscript. All authors read and approved the final manuscript.

\section{Acknowledgement}

The authors thank Ms Mihoko Inoue for manuscript preparation. This work was supported by Grants-in-Aid for Scientific Research from the Ministry of Education, Culture, Sports, Science, and Technology of Japan (Grant Numbers 21591289 and 24591472), Grants-in-Aid from The Japan Medical Association and Takeda Science Foundation.

Received: 14 March 2014 Accepted: 11 June 2014

Published: 11 July 2014

\section{References}

1. Stupi AM, Steen VD, Owens GR, Barnes EL, Rodnan GP, Medsger TA Jr: Pulmonary hypertension in the CREST syndrome variant of systemic sclerosis. Arthritis Rheum 1986, 29:515-524.

2. Ungerer RG, Tashkin DP, Furst D, Clements PJ, Gong H Jr, Bein M, Smith JW, Roberts N, Cabeen W: Prevalence and clinical correlates of pulmonary arterial hypertension in progressive systemic sclerosis. Am J Med 1983, 75:65-74.

3. MacLean MR: Endothelin-1: a mediator of pulmonary hypertension? Pulm Pharmacol Ther 1998, 11:125-132.

4. Channick RN, Simonneau G, Sitbon O, Robbins IM, Frost A, Tapson VF, Badesch DB, Roux S, Rainisio M, Bodin F, Rubin LJ: Effects of the dual endothelin-receptor antagonist bosentan in patients with pulmonary hypertension: a randomised placebo-controlled study. Lancet 2001, 358:1119-1123.

5. Rubin LJ, Badesch DB, Barst RJ, Galie N, Black CM, Keogh A, Pulido T, Frost A, Roux S, Leconte I, Michael L, Gérald S: Bosentan therapy for pulmonary arterial hypertension. N Engl J Med 2002, 346:896-903.

6. Barst RJ, Ivy D, Dingemanse J, Widlitz A, Schmitt K, Doran A, Bingaman D, Nguyen N, Gaitonde M, van Giersbergen PL: Pharmacokinetics, safety, and efficacy of bosentan in pediatric patients with pulmonary arterial hypertension. Clin Pharmacol Ther 2003, 73:372-382.

7. Humbert M, Segal ES, Kiely DG, Carlsen J, Schwierin B, Hoeper MM: Results of European post-marketing surveillance of bosentan in pulmonary hypertension. Eur Respir J 2007, 30:338-344. 
8. Sitbon O, Gressin V, Speich R, Macdonald PS, Opravil M, Cooper DA, Fourme T, Humbert M, Delfraissy JF, Simonneau G: Bosentan for the treatment of human immunodeficiency virus-associated pulmonary arterial hypertension. Am J Respir Crit Care Med 2004, 170:1212-1217.

9. Drug information in website of U.S. Food and Drug Administration, available from URL: www.accessdata.fda.gov/scripts/cder/drugsatfda/index.cfm.

10. Dingemanse J, van Giersbergen PL: Clinical pharmacology of bosentan, a dual endothelin receptor antagonist. Clin Pharmacokinet 2004, 43:1089-1115.

11. Fattinger K, Funk C, Pantze M, Weber C, Reichen J, Stieger B, Meier PJ: The endothelin antagonist bosentan inhibits the canalicular bile salt export pump: a potential mechanism for hepatic adverse reactions. Clin Pharmacol Ther 2001, 69:223-231.

12. Paolini M, Pozzetti L, Piazza F, Cantelli-Forti G, Roda A: Bile acid structure and selective modulation of murine hepatic cytochrome P450-linked enzymes. Hepatology 1999, 30:730-739.

13. Murdaca G, Spano F, Puppo F: Current therapies for the treatment of systemic sclerosis-related pulmonary arterial hypertension: efficacy and safety. Expert Opin Drug Saf 2014, 13:295-305.

doi:10.1186/1752-1947-8-250

Cite this article as: Ito et al:: Combined use of ursodeoxycholic acid and bosentan prevents liver toxicity caused by endothelin receptor antagonist bosentan monotherapy: two case reports. Journal of Medical Case Reports $20148: 250$

\section{Submit your next manuscript to BioMed Central and take full advantage of:}

- Convenient online submission

- Thorough peer review

- No space constraints or color figure charges

- Immediate publication on acceptance

- Inclusion in PubMed, CAS, Scopus and Google Scholar

- Research which is freely available for redistribution 